\title{
Effect of Low Dietary Glycemic Index on Blood Lipids Profile among Obese Postpartum Women
}

\author{
Shahnai Basharat, ${ }^{1}$ Syed Amir Gilani, ${ }^{2}$ Shahid Bashir, ${ }^{3}$ Muhammad Mustafa Qamar ${ }^{4}$
}

\begin{abstract}
Objective: Postpartum obesity leads to long-term maternal obesity and promotes drastic health complications. Low glycemic index diet is suggested to have a beneficial impact on blood lipid levels. Therefore, we conducted a study to explore the effect of low glycemic diet on blood lipid profile in obese postpartum women.
\end{abstract}

Methods: In a randomised controlled trial, 38 obese postpartum women in intervention and 36 obese postpartum women in control group were analysed. Subjects in the intervention group were assigned low glycemic index diet and to follow this protocol for 12 weeks and the control group was advised to continue their routine diet.

Results: Low glycemic diet had a positive impact on low density lipoprotein (LDL), triglyceride and high

\footnotetext{
${ }^{1}$ Assistant Professor, University Institute of Diet \& Nutritional Sciences, The University of Lahore

E-mail: shahnaibhatti@gmail.com

${ }^{2}$ Professor and Dean, Faculty of Allied Health Sciences The University of Lahore

${ }^{3}$ Head of Department, University Institute of Diet \& Nutritional

Sciences, The University of Lahore

${ }^{4}$ Assistant Professor, Medical College of Sargodha

University of Sargodha
}

Date of Submission: 14-02-2017

Date of Acceptance for Publication: 25-04-2017

Conflict of Interest: None

Funding Source: None

\section{Contribution}

All Authors have contributed in Study Design, Data Collection, Data Analysis, Data Interpretation, Manuscript Writing and Approval. density lipoprotein (HDL) concentration ( $\mathrm{p}<0.05)$. A strong positive correlation was observed between the glycemic index and LDL (mg/dl), $(r=0.57 ; \mathrm{p}=0.02)$ and between glycemic index and triglyceride $(\mathrm{mg} / \mathrm{dl})$, $(\mathrm{r}=0.51 ; \mathrm{p}=0.01)$ in control and intervention group. A negative correlation was identified between glycemic index and HDL $(\mathrm{mg} / \mathrm{dl}),(\mathrm{r}=-0.45 ; \mathrm{p}=0.01)$.

Conclusion: Study concluded that low glycemic index diet reduced low density lipoprotein and triglyceride level and increased HDL level in blood; further more a significant association was found between glycemic index and blood lipids profile.

Key Words: Low dietary glycemic index, Postpartum obesity, Blood lipids profile, Correlation.

\section{Introduction}

Obesity is increasing tremendously since 1980. In 2014, approximately $1.9 \times 10^{9}$ adults were obese, ${ }^{1}$ females were more vulnerable to obesity as compared with male with a ratio of $3: 2 .^{2}$ By 2015 , it was anticipated to rise $40 \%{ }^{3}$ According to Pakistan Demographic and Health Survey $\left(2012\right.$ - 2013), ${ }^{4} 40 \%$ women of 15 to 49 years were overweight or obese (BMI $\geq 25.0$ ). Moreover, increasing age also have a significant impact on weight. Only 7\% women were obese at the age of 19 years. However, $51 \%$ women were obese at the age of $40-49$ years. ${ }^{4}$

Childbearing years pose a high risk of obesity for women due to undue gestational weight gain (GWG) as well as postpartum weight retention (PPWR). These factors result in a poor health status of mothers and their infants. ${ }^{5}$ There is unavailability of adequately designed remedies for preventing and managing maternal obesity. ${ }^{6}$ Though, a few studies devised scientific diet to prevent/manage extra weight gain. Nutritional therapy is suggested a safe, risk-free and effective 
remedy to manage weight. ${ }^{7}$ In this specific approach, the key snacks are substituted by nutritionally balanced low-fat diet. ${ }^{8}$

In a study, ${ }^{9} 45$ total healthy overweight females within the age group of $20-40$ years were selected; the total length of the study was 10 weeks with 2 matched groups. Low glycemic index food was given to one group and the other group received high glycemic index foods. Study conclusion demonstrated significant decrease in body weight and fat mass, and up to $10 \%$ decreased in LDL and total cholesterol in low glycemic index group as compared to high glycemic index diet group. 9 A number of studies suggested that dietary glycemic index has an association with blood lipids profile. These studies showed that high glycemic index or glycemic load has adverse effects on blood lipid levels. ${ }^{10-12}$ Contrarily, in another study, ${ }^{13}$ the researcher examined the impact of low glycemic index (LGI) breakfasts on an overweight and obese individual and found no significant effect on lipid profile including triglycerides, HDL and LDL cholesterol concentration. $^{13}$

In the presence of equivocal findings, the effect of low glycemic index diet on blood lipids profile is still controversial. Therefore, aim of the study was to evaluate the impact of low glycemic index diet on blood lipids profile in postpartum obese women.

\section{Materials and Methods}

\section{Study Design and Subject Selection}

The randomised control Interventional trial was a part of large study project, examining the impact of low glycemic index diet on weight management in postpartum obese women. An Institutional Ethical Review Committee approved the study protocol and informed consent before study.

A brief overview of participant disposition is illustrated in Figure 1. 179 Primigravida obese postpartum women who were not practising breastfeeding selected and screened for induction in this study. The screening and data collection was accomplished with the help of a gynaecologist and clinical nutritionists of National Hospital and Medical Centre, Defence (DHA) Lahore and Services Hospital Lahore. 99 participants were excluded, among those 71 was not meet the study criteria and 28 reject to participate in after a demonstration of the study protocols. 80 participants qualified to include in this study. Physical Characteristics of participants illustrated in table 1 .
Table 1: Physical characteristic of the participants.

\begin{tabular}{|l|c|}
\hline Age (Years) & $27 \pm 5$ \\
\hline Height $(\mathrm{CM})$ & $160 \pm 1.2$ \\
\hline Weight $(\mathrm{KG})$ & $80 \pm .4$ \\
\hline BMI & $31.3 \pm 1.5$ \\
\hline
\end{tabular}

The participants were randomly recruited into Control group $(\mathrm{n}=40)$ and Interventional group $(\mathrm{n}=40)$. The control group used routine diet as before, however, Interventional group had specifically designed diet protocol, including low glycemic diet for 12 weeks. Participants discontinued all dietary supplements and weight loss medication/procedures.

Postpartum weight retention comprised pre-pregnancy weight (at the time of conception), and gestational weight gain, and then weight at delivery was assessed as "the sum of these two weights. Weight loss from birth to baseline was estimated as weight at baseline minus weight at delivery". ${ }^{14}$ Eligibility criteria was: available weight data pre-pregnancy and baseline $\mathrm{BMI} \geq 30 \mathrm{~kg} / \mathrm{m}^{2}$, and women having given birth within the last six weeks.

The postpartum weight retention was then calculated by 6 weeks postpartum $=$ Pre-pregnancy weight weight at baseline. ${ }^{5}$ Pre-pregnancy obese women and those who suffered from uncontrolled hypertension, diabetes mellitus, cardiovascular, endocrine disease and cancer before and at the time of screening were excluded.

\section{Dietary Intervention and Assessment}

Foods with a Glycemic Index (GI) $<55$ considered as low GI foods that prescribed to intervention group by following 7 - days menu cycle, while the control group used normal routine diet. Data from 24 - hour recall was used to calculate total Kcal, macronutrient intake, total glycemic index with the help of using food-composition tables, ${ }^{15}$ International table of glycemic index and glycemic load, ${ }^{16}$ and glycemic index of Indian rotis. ${ }^{17}$ Total dietary glycemic index was calculated by multiply the amount of available carbohydrate (g) of each food item by that food glycemic index. Then the sum of these products was divided by total carbohydrate intake. ${ }^{18}$ 


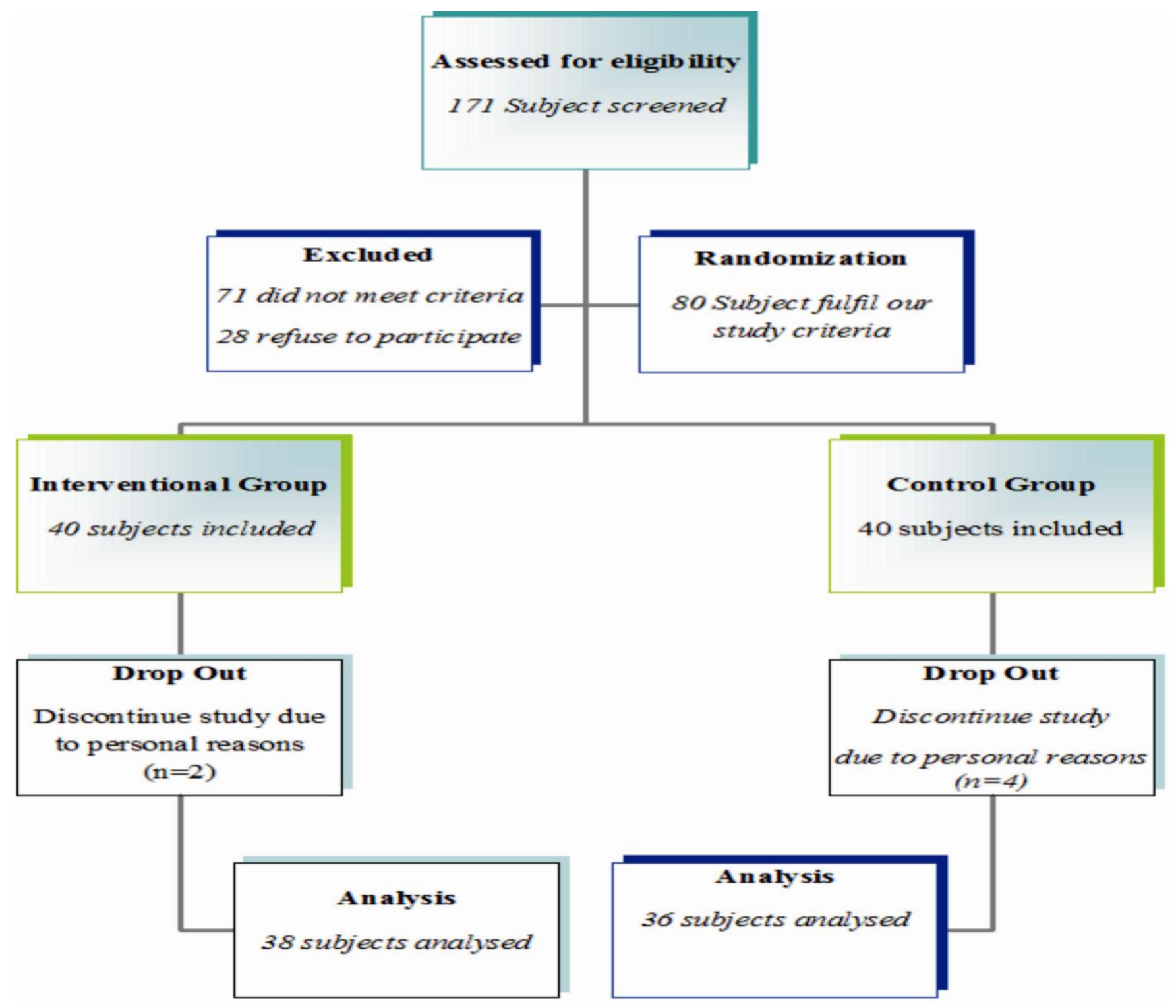

Fig. 1: A Brief Overview of the Participant's Disposition.

\section{Data Collection Procedure}

The study included following clinical visits of both groups; 1) Visit for screening for application of inclusion or exclusion criteria; 2) Visit for baseline measurement 3) One visit after each four week for supervision during weight loss treatment for 12 weeks of intervention 4) Visit for post-study measurements.

At the baseline screening visit, the data was collected within six weeks of postpartum included baseline biochemical evaluation and dietary intake data estimated by using 24 - hour dietary recall and food frequency table (per week). At final visit, post study measurement was taken.

\section{Statistical Analysis}

Statistical analysis was conducted by using SPSS software version 18 (SPSS Inc., Chicago, IL), personal computer was used for storage of data. Quantitative data was demonstrated as mean, standard deviation. The unpaired two-tailed t-test was used to analyze the differences in parameters at baseline and at post study time between interventional and control group and Pearson correlation coefficient used for evaluating the association between glycemic index and biochemical evaluation. A p value $<0.05$ was considered as statistically significant. 


\section{Results}

\section{Comparison between Control and Intervention Group at Baseline}

No statistically significant differences were found in biochemical evaluation parameters between the baseline values of the control and intervention groups (Table 1).

Table 1: Biochemical Evaluation in Control and Intervention Group at Baseline.

\begin{tabular}{|l|c|l|c|}
\hline & $\begin{array}{c}\text { Control } \\
\text { Group }\end{array}$ & $\begin{array}{c}\text { Intervention } \\
\text { Group }\end{array}$ & $\begin{array}{c}\text { P- } \\
\text { Value }\end{array}$ \\
\hline LDL mg/dl & $118.2 \pm 1.2$ & $119 \pm 1.5$ & 0.89 \\
\hline HDL mg/dl & $46.2 \pm 2.1$ & $48.1 \pm 1.1$ & 0.95 \\
\hline $\begin{array}{l}\text { Triglyceride } \\
\text { mg/dl }\end{array}$ & $156.1 \pm 1.8$ & $155.3 \pm 10.1$ & 0.75 \\
\hline
\end{tabular}

Changes in Biochemical Evaluation after 12 Weeks in Obese Post-partum Women

No changes were seen in biochemical evaluation at the end of 12 weeks in the control group. However, the intervention group showed significant changes in LDL level changes from $119 \pm 1.5$ to $111 \pm 1.2 \mathrm{mg} / \mathrm{dl}$ after the study period, and changes were seen in HDL level from $48.1 \pm 1.1$ to $52.02 \pm 1.21 \mathrm{mg} / \mathrm{dl}$. Interestingly, remarkable changes were established in Triglyceride after 12 weeks of the study period (Table 2).

\section{Relationship between the Glycemic Index, LDL} (mg/dl), HDL (mg/dl) and Triglyceride (mg/dl)

A strong positive correlation was observed between the glycemic index and LDL $(\mathrm{mg} / \mathrm{dl}),(\mathrm{r}=0.57 ; \mathrm{p}=$ $0.02)$ and between glycemic index and Triglyceride $(\mathrm{mg} / \mathrm{dl}),(\mathrm{r}=0.51 ; \mathrm{p}=0.01)$ in control and intervention group. On the other hand, a negative correlation was found between glycemic index and HDL $(\mathrm{mg} / \mathrm{dl}),(\mathrm{r}=$ $-0.45 ; \mathrm{p}=0.01),($ Graph 1, 2, 3).

Table 2: Biochemical Evaluation in Control and Intervention Group at Post Study Time.

\begin{tabular}{|l|c|c|c|c|c|}
\hline \multirow{2}{*}{} & \multicolumn{2}{|c|}{ Control Group } & \multicolumn{2}{c|}{ Intervention Group } & \multirow{2}{*}{ P-Value } \\
\cline { 2 - 5 } & Baseline & Post Study & Baseline & Post Study & \\
\hline LDL mg/dl & $118.2 \pm 1.2$ & $123 \pm 2.5$ & $119 \pm 1.5$ & $111 \pm 1.2$ & 0.003 \\
\hline HDL mg/dl & $46.2 \pm 2.1$ & $44.3 \pm 1.1$ & $48.1 \pm 1.1$ & $52.02 \pm 1.21$ & 0.002 \\
\hline Triglyceride mg/dl & $156.1 \pm 1.8$ & $162 \pm 2.1$ & $155.3 \pm 10.1$ & $146.5 .5 \pm 1.51$ & 0.02 \\
\hline
\end{tabular}

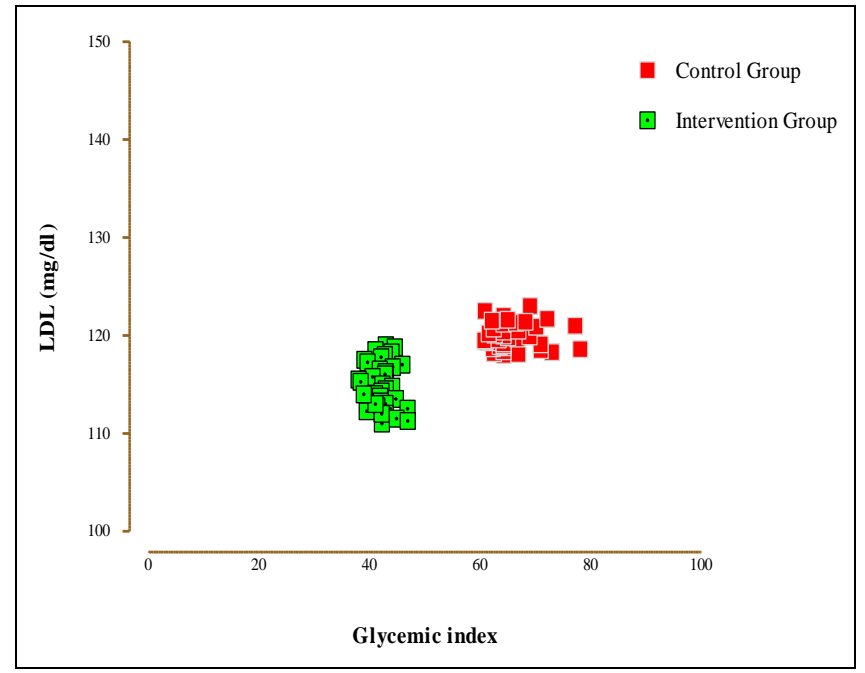

Graph 1: Relationship between the Glycemic Index and $L D L(m g / d l)$ in Control and Intervention Group; $r=0.57 ; p=0.02$.

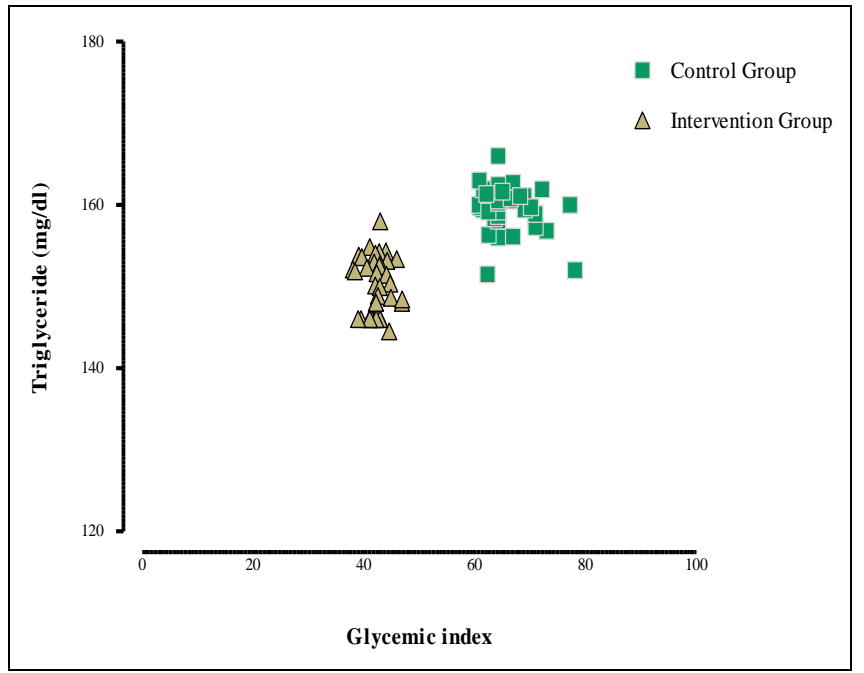

Graph 2: Relationship between the Glycemic Index and Triglyceride $(\mathrm{mg} / \mathrm{dl})$ in Control and Ntervention Group; $r=0.51 ; p=0.01$ 


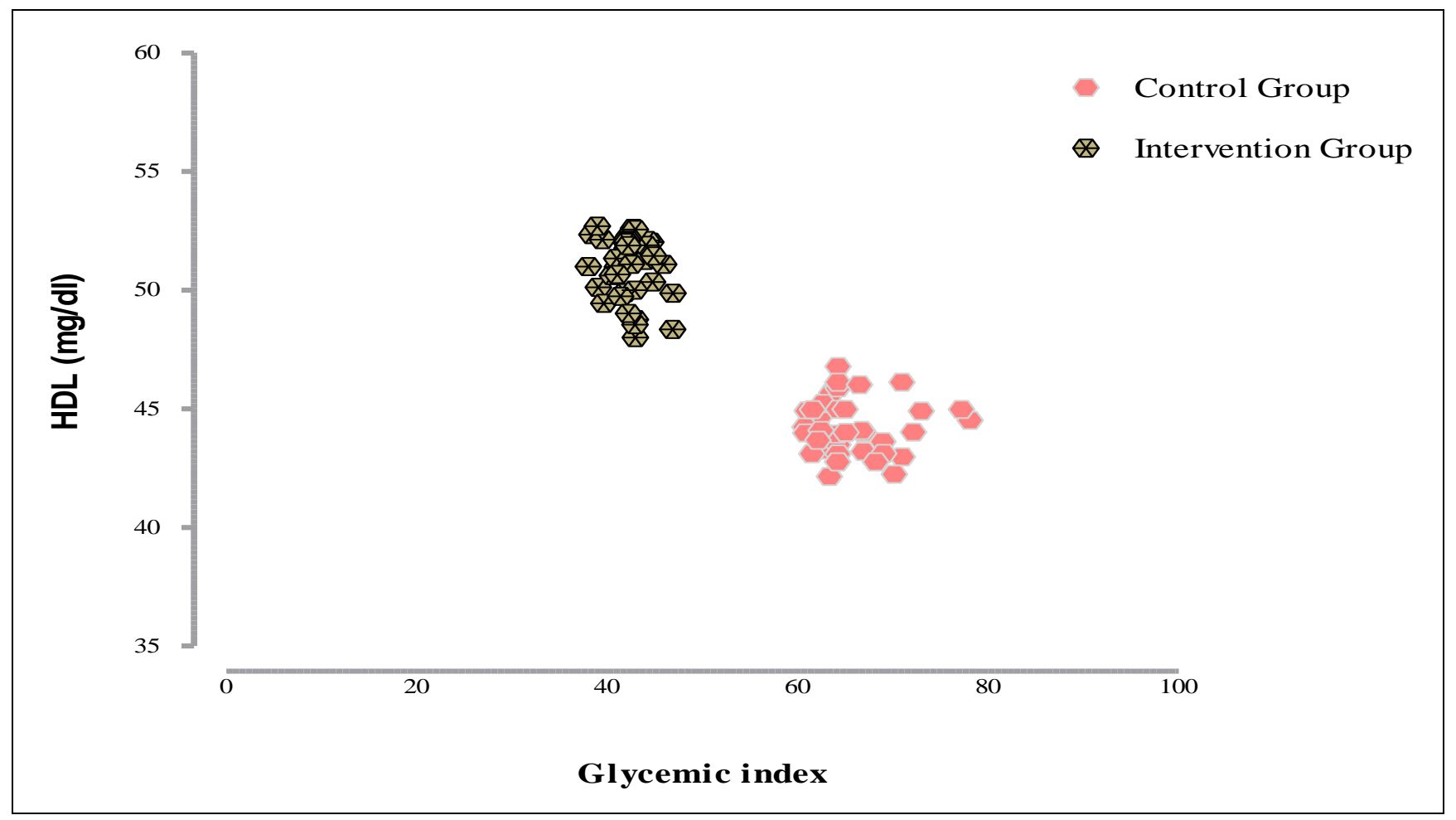

Graph 3: Relationship between the Glycemic Index and HDL $(\mathrm{mg} / \mathrm{dl})$ in Control and Intervention Group; $r=-0.45$; $p=0.01$.

\section{Discussion}

To researcher knowledge, this is the prime research in Pakistan to explore the effect of low glycemic index diet on biochemical markers among obese postpartum women. The main findings were a significant improvement in biochemical marker after 12 weeks in intervention group. Moreover, a strong association was found between low glycemic index and blood lipids profile. A strong positive correlation was observed between the glycemic index and LDL, Triglyceride level and a negative correlation was found between glycemic index and HDL level.

Results of the study suggested that the blood concentration of triglyceride and LDL increased in the control group, having a high glycemic index food intake (64.23 \pm 46.34$)$. On the other hand, in intervention group blood concentration of triglyceride and LDL significantly decreased, having low glycemic index $\operatorname{diet}(42.95 \pm 0.88)$.

Studies suggested that dietary glycemic index and glycemic load has an association with blood lipids level. ${ }^{10-12,19,20}$ These studies showed that high glycemic index or glycemic load has noxious effects on blood lipid levels. It had been analysed high glycemic index or low to high quintiles of glycemic load associated with elevated triglyceride levels and Low density lipoprotein level, ${ }^{11,12}$ and having a inverse correlation between high density lipoprotein concentration and dietary glycemic index or glycemic load. ${ }^{11,12,21-23}$

Miller $^{24}$ reviewed eleven short and long period studies that examine glycemic index and blood lipid management. Most of 1 week to 3 months' studies showed that reduced $>12$ units of overall dietary glycemic index help to reduce mean $9 \%$ of triglyceride concentration. ${ }^{24} \mathrm{~A}$ meta-analysis of 14 randomised controlled trials was conducted by Opperman et al. ${ }^{25}$ to relate the efficacy of altering glycemic index of test diets on blood lipids and analysed low GI diet elevate HDL concentration and vice versa for LDL and triglyceride blood levels with high GI diet. ${ }^{25}$

Present study results are found compatible with the results found by Gutierrez and his collaborates. ${ }^{10}$ In the present study, intervention group participants with low glycemic index showed reduction in triglyceride concentration and LDL concentration (155.3 \pm 10.1 to $146.5 .5 \pm 1.51 \mathrm{mg} / \mathrm{dl} ; 119 \pm 1.5$ to $111 \pm 1.2$ $\mathrm{mg} / \mathrm{dl})$ respectively, and an increase in high-density lipoprotein (HDL) $(48.1 \pm 1.1$ to $52.02 \pm 1.21 \mathrm{mg} / \mathrm{dl})$. 
In control group, a high glycemic index significantly associated increase in triglyceride and LDL blood levels $(156.1 \pm 1.8$ to $162 \pm 2.1 \mathrm{mg} / \mathrm{dl} ; 118.2 \pm 1.2$ to $123 \pm 2.5 \mathrm{mg} / \mathrm{dl})$ and decrease in HDL level $(46.2 \pm$ 2.1 to $44.3 \pm 1.1 \mathrm{mg} / \mathrm{dl})$, (Table 2$)$.

As nutrition plays a vital role in improving health quality and disease prevention. The study provides insight to explore the effect of low glycemic index diet on blood lipids profile among obese postpartum women. Many foods are available in the market with the low glycemic index but people do not have enough knowledge to select and use these foods. The study has provided well balanced low glycemic index food plan for the reduction of obesity, low-density lipoprotein, and triglyceride lipids because increased low-density lipoprotein and triglyceride concentration can be a leading cause of cardiovascular diseases and even death.

In conclusion, this study adds growing evidence; that low glycemic index diet improves high-density lipoprotein level and lowered low-density lipoprotein and triglyceride concentration in blood profile.

\section{References}

1. World Health Organization. Obesity and overweight: Fact sheet 2015. Available from: http://www.who.int/mediacentre/factsheets/fs311/en/. Accessed.2015.

2. Tjepkema M. Adult obesity in Canada: measured height and weight Nutrition Findings from Canadian Community Health Survey, 2008. Available from http://www.statcan.gc.ca/pub/82-620$\mathrm{m} / 2005001 /$ article/adults-adultes/8060eng.htm. Accessed. 2015.

3. World Health Organization. Core Health Indicators. 2011. Available from:

http://apps.who.int/whosis/database/core/core_select.cf m. Accessed.2015.

4. National Institute of Population Studies and ICF International. Pakistan Demographic and Health Survey, 2012-13.2013. Available from: http://www.nips.org.pk/abstract_files/Priliminary\%20R eport\%20Final.pdf. Accessed Mar 15, 2015.

5. Gunderson EP, Abrams B, Selvin S. The relative importance of gestational gain and maternal characteristics associated with the risk of becoming overweight after pregnancy. Int J Obes Relat Metab Disord. 2000; 24 (12): $1660-8$.

6. Birdsall KM, Vyas S, Khazaezadeh N. Maternal obesity: a review of interventions. Int J Clin Pract. 2009; 63 (3): 494-507.

7. Davis LM, Coleman C, Kiel J. Efficacy of a meal replacement diet plan compared to a food-based diet plan after a period of weight loss and weight maintenance: a randomized controlled trial. Nutr J. 2010; 9 (1): 11.

8. LeCheminant JD, Jacobsen DJ, Hall MA. A comparison of meal replacements and medication in weight maintenance after weight loss. J Am Coll Nutr. 2005; 24 (5): 347-53.

9. Sloth B, Krog-Mikkelsen I, Flint A. No difference in body weight decrease between a low-glycemic-index and a high-glycemic-index diet but reduced LDL cholesterol after 10-wk ad libitum intake of the lowglycemic-index diet. Am J Clin Nutr. 2004; 80 (2): 337-47.

10. Denova-Gutierrez E, Huitron-Bravo G, Talavera JO. Dietary glycemic index, dietary glycemic load, blood lipids, and coronary heart disease. J Nutr Metab. 2010.

11. Amano Y, Kawakubo K, Lee J. Correlation between dietary glycemic index and cardiovascular disease risk factors among Japanese women. Eur J Clin Nutr. 2004; 58 (11): 1472-78.

12. Liu S, Manson JE, Stampfer MJ. Dietary glycemic load assessed by food frequency questionnaire in relation to plasma high-density-lipoprotein cholesterol and fasting plasma triacylglycerols in postmenopausal women. Am J Clin Nutr. 2001;73 (3): 560-6.

13. Pal S, Lim S, Egger G. The effect of a low glycaemic index breakfast on blood glucose, insulin, lipid profiles, blood pressure, body weight, body composition and satiety in obese and overweight individuals: a pilot study. J Am Coll Nutr. 2008; 27 (3): 387-93.

14. Wiltheiss GA, Lovelady CA, West DG. Diet quality and weight change among overweight and obese postpartum women enrolled in a behavioral intervention program. J Acad Nut Diet. 2013; 113 (1): 54-62.

15. Whitney E, Rolfes SR. Understanding nutrition. 13th ed. USA: Cengage Learning, 2013.

16. Atkinson F, Foster-Powell K, Brand-Miller JC. International table of glycemic index and glycemic load values: 2008. Diabetes care, 2008; 31 (12): 2281-83.

17. Radhika G, Sumathi C, Ganesan A. Glycaemic index of Indian flatbreads (rotis) prepared using whole wheat flour and 'atta mix'-added whole wheat flour. $\mathrm{Br} \mathrm{J}$ Nutr. 2010; 103 (11): 1642-47.

18. Wolever TM, Nguyen P-M, Chiasson J-L. Relationship between habitual diet and blood glucose and lipids in non-insulin dependent diabetes (NIDDM). Nutrition Research, 1995; 15 (6): 843-57.

19. Levitan EB, Cook NR, Stampfer MJ. Dietary gly-cemic index, dietary glycemic load, blood lipids, and C-reactive protein. Metabolism, 2008; 57 (3): 437-43.

20. Liu S, Manson JE, Buring JE. Relation between a diet with a high glycemic load and plasma concent-rations of high-sensitivity C-reactive protein in middle-aged women. Am J Clin Nutr. 2002; 75 (3): 492-98.

21. Ford ES, Liu S. Glycemic index and serum high-density lipoprotein cholesterol concentration among US adults. 
Arch Intern Med. 2001; 161 (4): 572-76.

22. Mensink RP, Zock PL, Kester AD. Effects of die-tary fatty acids and carbohydrates on the ratio of serum total to HDL cholesterol and on serum lipids and apo-lipoproteins: a meta-analysis of 60 controlled trials. Am J Clin Nutr. 2003; 77 (5): 1146-55.

23. Slyper A, Jurva J, Pleuss J. Influence of glycemic load on HDL cholesterol in youth. Am J Clin Nutr. 2005; 81 (2): 376-9.

24. Miller JC. Importance of glycemic index in diabetes. Am J Clin Nutr. 1994; 59 (3): 747-52.

25. Opperman AM, Venter CS, Oosthuizen W. Meta-analysis of the health effects of using the glycaemic index in meal-planning. Br J Nutr. 2004; 92 (3): 367-81. 